\title{
"The Flipping Bullet" with Associated Intramedullary Dystrophic Calcification: An Unusual Cause for Migratory Myelopathy and Radiculopathy
}

\author{
Christopher H. Hunt ${ }^{*}$, Gavin A. McKenzie, Felix E. Diehn, Jonathan M. Morris, Christopher P. Wood
}

Mayo Clinic, Department of Radiology Rochester, Minnesota

\begin{abstract}
We report the case of a 24 year old male who had a retained bullet within his thoracic spine from a gunshot wound resulting in paraplegia. After 7 months he began experiencing painful dysesthesias at his sensory level. Repeat imaging demonstrated migration of the bullet as well as the development of intramedullary dystrophic calcification associated with the bullet. This case demonstrates not only the ability for retained bullets to migrate within the spinal canal but also demonstrates they can lead to remote symptoms due to the development of dystrophic calcification.
\end{abstract}

Keywords: Bullet, dystrophic calcification, spinal cord trauma.

\section{INTRODUCTION}

Bullet migration within the thecal sac has been described in several case reports resulting in a spectrum of presentations, ranging from no change in symptoms to increased myelopathy [1]. While the metallic composition of the bullet should be relatively biologic inert, remote neurologic deterioration without physical migration has raised concerns in some patients of possible granulomatous or dystrophic reactions [2]. We present a case that demonstrates not only bullet migration within the thecal sac leading to increased myelopathy, but also provides imaging evidence of remote intramedullary dystrophic reaction to the bullet.

\section{CASE REPORT}

A 24 year-old male was shot in the left shoulder after an altercation. The $15 \mathrm{~mm}$ bullet trajectory was carrying it towards the mediastinum when it ricocheted off the left sixth rib and traveled into the thoracic spinal canal via a neural foramen where it became lodged at the T8-9 interspace level. Upon admission, the patient was intoxicated, but otherwise appeared to have a normal mental status. He had a normal cranial nerve and upper extremity motor and sensory exam, but a flaccid paraplegia of the lower extremities with a dense sensory level at T8.

Initial noncontrast $\mathrm{CT}$ scan performed at the time of the trauma revealed the unfractured bullet was pointing cephalad within the spinal cord at the T8-T9 interspace, correlating with the patient's neurologic examination (Fig. 1A). As the patient had such dense paraplegia and there was no associated spinal fracture, the decision was made to treat him non-operatively. Once stabilized over the next week, he was transferred to inpatient rehabilitation. Approximately 7

*Address correspondence to this author at the Mayo Clinic, Department of Radiology 200 First Street SW Rochester, Minnesota 55905; Tel: 507-284-5114; Fax: 507-293-3680;

E-mail: hunt.christopher@mayo.edu months later, he began experiencing painful dysesthesias in a band like region around his lower chest and upper abdomen without any change in his dense paraplegia. Given the painful nature of his complaints as well as the change in his symptomatology, a repeat CT of the thoracic spine was performed. Repeat CT revealed the bullet had migrated inferiorly to the T9-10 interspace and had "flipped" with the tip now pointed caudally (Fig. 1B). A repeat neurosurgical consultation suggested possibly removing the "flipping" bullet, however, given the patients stable motor exam, the decision was made by the patient and medical team to observe only. Repeat CT at one year demonstrated the bullet in the same position without further change in orientation. There was now, however, associated dystrophic intramedullary calcification extending from both ends of the bullet from T9 to T11 (Fig. 1C). This calcification was felt to be reactive. There was significant concern about the possibility of the dystrophic calcification extending more cranially and leading to progressive motor deterioration. However, given that the patient's painful migratory radiculopathy symptoms had abated over the interim without new complaints, the decision was made to continue to observe and not to surgically intervene.

\section{DISCUSSION}

Besides being due to the immediate penetrating trauma, neurologic deterioration from retained intradural bullet fragments is exceedingly rare with only limited case reports available in the literature [2]. Asymptomatic bullet migration incidentally noted on other studies or at followup appears to be more common [1, 3, 4], Likely mediated by gravitational forces as well as by pressure gradients created by coughing, swallowing, and cerebrospinal fluid flow, migration is typically in the caudal direction. While sudden movement of bullets within the thecal sac can lead to acute compressive syndromes, slowly progressive deterioration has also been documented and ascribed to progressive epidural fibrosis with or without progressive degenerative disease at the level of the trapped bullet [2]. 


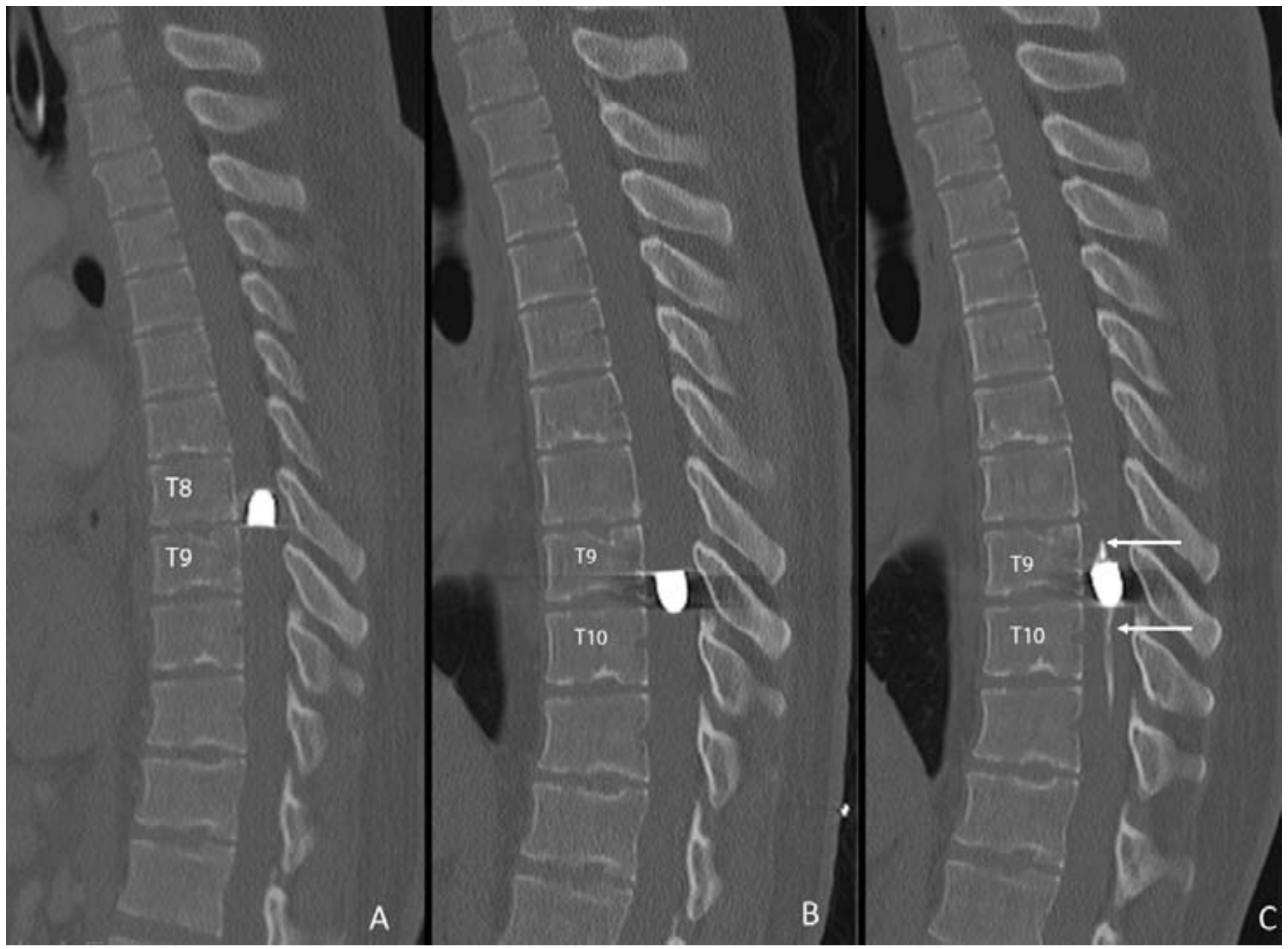

Fig. (1). Noncontrast CT reconstructed sagittal images of the thoracic spine. At initial presentation (A), the bullet within the spinal canal was pointed cephalad at the T8-9 interspace. Seven months later (B), due to progressive symptoms, repeat imaging revealed the bullet had "flipped" with tip now pointed caudally. In addition, the bullet had migrated inferiorly to the T9-T10 interspace. One year after the original trauma $(\mathbf{C})$, the bullet had remained stable in position since prior exam, but now had developed worrisome intramedullary dystrophic calcification (arrows) extending both cranially and caudally.

Our case suggests that in some patients there is the potential for neurologic progression from both movement of the bullet as well as from a reactive process associated with the bullet itself, suggesting that they cannot always be assumed to be biologically inert. In fact, dystrophic calcification associated with retained foreign bodies is not infrequent. The dystrophic calcification in our case was likely due to formation of biologic hydroxyapatite on the surface of the bullet due to heterogenous nucleation. This process is commonly seen at metallic interfaces, most commonly due to orthopedic implants [5]. In our patient, we initially assumed that the new sensory dysesthesias were due to the inferior migration as well as the "flipping" of the bullet. In patients who were more neurologically intact than our patient, this caudal translation of the bullet could have had more serious consequences. On further followup though, the reactive component of the bullet retained within the thecal sac was seen. The intramedullary dystrophic calcification that encased and extended both cranially and caudally from the bullet was alarming. Concern that continued cranial extension of this process could lead to worsened motor status led to the consideration for removal of the bullet. While the decision was made ultimately to observe this patient, continued neurologic examinations and imaging surveillance are planned to ensure stability.

Intramedullary dystrophic ossification has been reported in post-traumatic and post-surgical cases; [6] however, we believe that this is the first example of its association with a retained bullet within the spine. In these post-traumatic or post-surgical cases, this dystrophic ossification is usually a marker for poor outcome. While it is hard to generalize on the basis of this single case, in patients with remote neurologic deterioration with a retained intradural bullet, repeat CT imaging and consideration of surgical removal should at least be considered to potentially prevent further progression. If removal of the bullet is decided against, careful surveillance for imaging stability both in terms of positions, orientation, and dystrophic changes is recommended, as this may alter clinical and surgical management decisions.

\section{CONFLICT OF INTEREST}

The author confirms that this article content has no conflicts of interest. 


\section{ACKNOWLEDGEMENT}

Declared none.

\section{REFERENCES}

[1] Farrugia A, Raul J-S, Geraut A, Ludes B. Ricochet of a bullet in the spinal canal: a case report and review of the literature on bullet migration. J Forensic Sci 2010; 55: 1371-4.

[2] Ajmal S, Enam SA, Shamim MS. Neurogenic claudication and radiculopathy as delayed presentations of retained spinal bullet. Spine J 2009; 9: e5-8.
[3] Cagavi F, Kalayci M, Seckiner I, et al. Migration of a bullet in the spinal canal. J Clin Neurosci 2007; 14: 74-6.

[4] Karim NO, Nabors MW, Golocovsky M, Cooney FD. Spontaneous migration of a bullet in the spinal subarachnoid space causing delayed radicular symptoms. Neurosurgery 1986; 18: 97-100.

[5] Pham MT, Maitz MF, Matz W, Reuther H, Richter E, Steiner G. Promoted hydroxyapatite nucleation on titanium ion-implanted with sodium. Thin Solid Films 2000; 379: 50-6.

[6] Lawson-Smith M, Green AL, Teddy PJ. Cord compression secondary to intradural ossification. J Clin Neurosci 2006; 13: 2725 .

Received: June 29, 2012

Revised: August 07, 2012

Accepted: August 09, 2012

(C) Hunt et al.; Licensee Bentham Open.

This is an open access article licensed under the terms of the Creative Commons Attribution Non-Commercial License (http://creativecommons.org/licenses/by$\mathrm{nc} / 3.0 /$ ), which permits unrestricted, non-commercial use, distribution and reproduction in any medium, provided the work is properly cited. 